\title{
Therapeutic Effect of Chemically Synthesized Silver Nanoparticles on Aphthous Stomatitis in Some Iraqi Patients
}

\author{
Zahraa Neamah Abbas ${ }^{1}$, Nafal NazarBahjat ${ }^{2}$ \\ ${ }^{1}$ Assistant Lecturer, ${ }^{2}$ Lecturer, department of Medical Analysis, College of Health and Medical Technologies, \\ Middle Technical University, Baghdad-Iraq
}

\begin{abstract}
Silver nanoparticles (AgNPs) were synthesized by a chemical reduction method. The particles were synthesized using silver nitrate solution with sodium borohydride as the reducer. The resulting colloidal AgNPs were characterized by UV-Visible spectroscopy, and FTIR. Aphthous stomatitis is a common and recurrent painful disease of the oral mucous membrane, classified in to three different aphthous types: minor, major and herpetiform. It may appear due to a variety of causes including: stress, food, hypersensitivities, infection, trauma, drug allergy, nutrition deficiencies (Iron, zinc \& vitamin B12) or it could be a symptom of serious disease such as carcinoma. In this study, (75) young Iraqi patients aged (18-24) years divided in to two groups according to their gender ( 25 male and 50 female) all of them are suffering from major Aphthous ulcers in the mouth. The solution of $(10 \mu \mathrm{g} / \mathrm{ml})$ concentration of silver nanoparticles showed a uniform cycle inhibition around the ulcer growth zone while, increasing of (AgNPs) solution to $(20 \mu \mathrm{g} / \mathrm{ml})$ showed a highly and significant inhibitory effect against isolates from Aphthous stomatitis during the first 24 hours only in both male and female groups. Further studies is needed to investigate the inhibitory mechanism of (AgNPs) against Aphthous stomatitis.
\end{abstract}

Keywords: patients; health; therapy; toxicity; Silver NPs; Aphthous

\section{Introduction}

A great attention has been directed toward the use of new medicinal preparations at nanoscale levels. A huge number of physical, chemical, biological and hybrid methods are available to synthesis different types of nanoparticles (NPs) ${ }^{(1)}$. Silver nanoparticles (AgNPs) of $100 \mathrm{~nm}$ dimension or less have unique physical, chemical and biological properties ${ }^{(2)}$. AgNPs are considered one of the most promising anticancer agents, protects against bacterial $^{(2)}$, fungal ${ }^{(3)}$, and viral infections ${ }^{(4)}$.

Aphthous stomatitis is a common and recurrent painful disease of the oral mucous membrane. It is start as small red and simple or grouped papules, classified in to three different aphthous types: minor, major and herpetiform. It may appear due to a variety of causes

\section{Correspond author:}

Zahraa Neamah Abbas

E-mail: zah.gas80@gmail.com including: stress, food, hypersensitivities, infection, trauma, drug allergy, nutrition deficiencies (Iron, zinc \& vitamin B12) or it could be a symptom of serious disease such as carcinoma ${ }^{(1)}$.

Minor aphthus stomatitis usually occurs in crops of 1-5. They are small; usually less than $1 \mathrm{~cm}(5 \mathrm{~mm})$ in diameter appears as a white or yellowish center with an inflamed red out edge, roundest, painful \& shallow. Common sites are tongue margin \&inside the lips \&cheeks normally take 7-14 days to heal. While major aphthous stomatitis characterized by large (greater than $1 \mathrm{~cm}$ in diameter), numerous in crops of 10 or more. It heals slowly \& may persist for many weeks. Herpetiform (uncommon) are pinpoint $\&$ occur in large crops of up to 100 at a time, usually, heal within a month, often occur in posterior part of mouth (an unusual location for MAU) ${ }^{(5)}$.

However, many cases may associate with severe pain and difficulty in eating thus a topical corticosteroid and anesthetic can provide a short-term relief and facilities 
eating ${ }^{(5)}$. Silver nitrate is often used as a chemical cautery, while carbon dioxide laser can provide pain relief almost instantaneously ${ }^{(1)}$.

Actually, there is no effective medication or treatment for the prevention or even future recurrence. The aim of our study is to synthesize colloidal silver nanoparticles and exert their effects on the recurrent aphthous stomatitis.

\section{Material and Method}

\section{A) Material}

Silver Nitrate $\left(\mathrm{AgNO}_{3}\right)$, sodium borohydride $\left(\mathrm{NaBH}_{4}\right)$ merck (Germany), Mueller Hinton Agar was procured from Himedia (India). Sterile distilled water was used throughout the experiments.

B) Chemical synthesis of silver nanoparticles

Oxidation reduction method was used for the synthesis of silver nanoparticles, $0.01 \mathrm{M}$ of sodium borohydride was added drop by drop to $0.001 \mathrm{M}$ of silver nitrate solution under stirring. Color changing is appearing from pale yellow, red and finally to almost black color as shown in figure 1. Centrifuged the resulted aqueous solution at $1500 \mathrm{rpm}$ for 30 minutes and dried at $100{ }^{\circ} \mathrm{C}^{(6)}$.

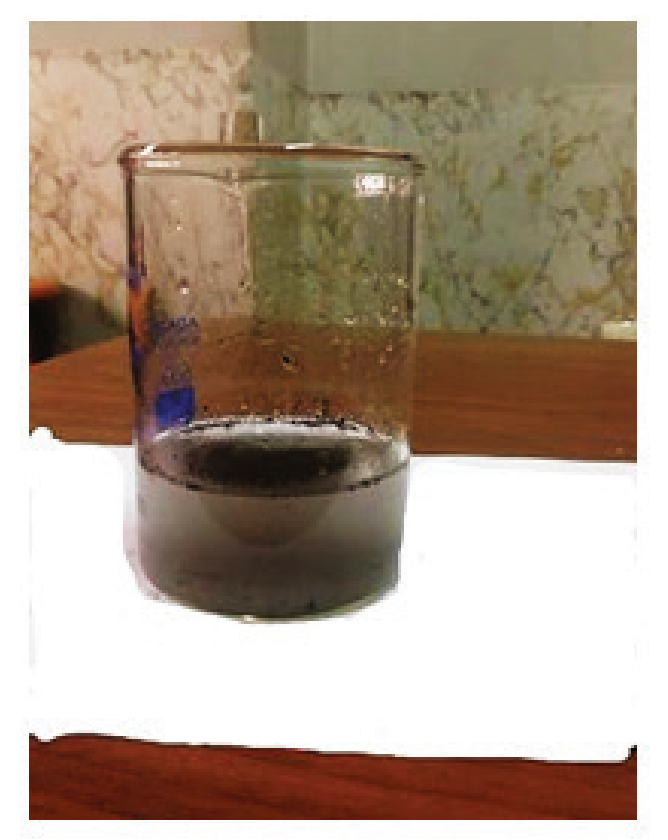

Figure (1): Synthesized colloidal silver nanoparticle solution

\section{C) Sample collection}

Seventy-five young patients ( 25 male and 50 female) aged 18-24 years suffering from Aphthous ulcers in the mouth arrived to biology lab, department of Medical Analysis College of Health and Medical Technologies, Middle Technical University, Iraq. Clinical samples were collected by sterile swops for major and severe aphthous stomatitis only, minor aphthous stomatitis and simple cases were excluded.

D) Silver nanoparticles test

A solution of two different concentrations of Silver nanoparticles were tested for their activity against identified aphthous ulcer by the modified Kirby Bauer agar well diffusion method according to the CLSI guidelines $(2011)^{(7)}$. The preferred concentration 10 , $20, \mu \mathrm{g} / \mathrm{ml}$ were prepared and twenty milliliter of nutrient agar medium was poured into sterilized Petri plates. With the help of a sterilized borer, agar wells of $6 \mathrm{~mm}$ diameter were prepared. The wells were injected with $10,20 \mu 1$ of the synthesized silver nanoparticle solution, $20 \mu \mathrm{l}$ of $1 \mathrm{mM} \mathrm{AgNO}_{3}$ as a negative control. The plates were further incubated at $37{ }^{\circ} \mathrm{C}$ for $24 \mathrm{~h}$ and were then examined for the presence of zones of inhibition. The inhibition zone was measured and expressed in centimeter scale $^{(6)}$.

\section{Results and Discussion}

A) Fourier transform infrared spectroscopy FTIR

FTIR analyses were carried out using ALPHA FTIR spectrophotometer figure (2). The samples were prepared by using the $\mathrm{KBr}$ pellets technique and were analyzed to check the presence of functional groups of the surface chemistry of the reduced (AgNPs). The FTIR spectrums were collected at a spatial resolution of $4 \mathrm{~cm}^{-1}$ in the transmission mode, between 4000 and $400 \mathrm{~cm}^{-1}$, respectively ${ }^{(8)}$. 


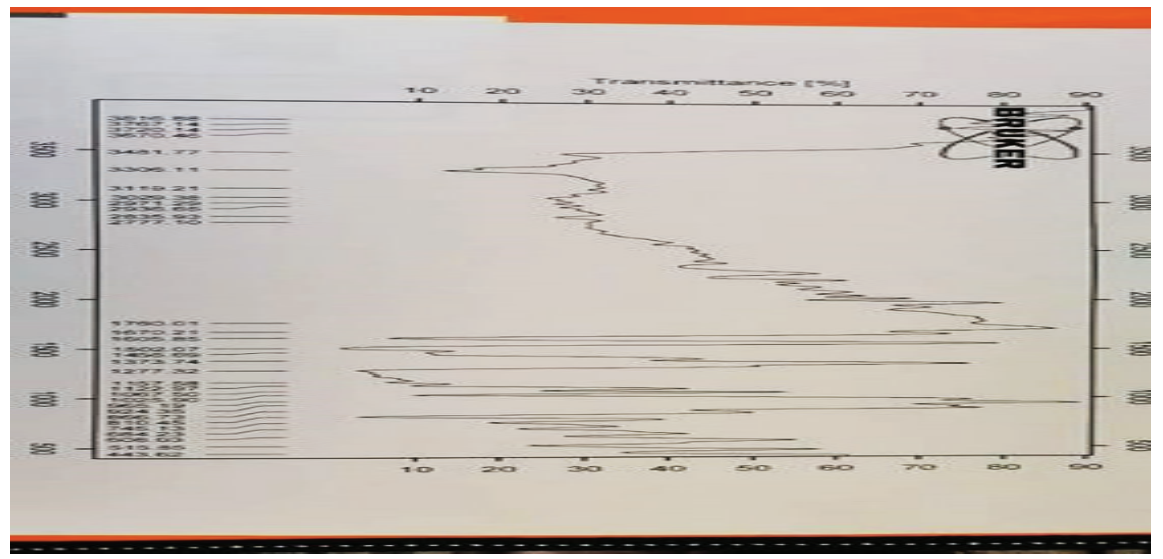

Figure (2): FTIR spectrum of chemically synthesized silver nanoparticles

\section{B) Silver nanoparticles activity}

Recurrent aphthous stomatitis (RAS) commonly known as aphthae or canker sores, have uncertain etiology with causes not fully elucidated ${ }^{(9)}$. Minor (RAS) are mostly small and below $5 \mathrm{~mm}$ in diameter that heal between 4-7 days and usually no treatment is needed, whereas, the severe painful ulcers may be debilitating causing weight loss due to malnutrition.

To our knowledge, there is no published study on the effect of silver nanoparticles in aphthous ulceration. The current study included (75) young Iraqi patients aged 18-24 years divided in to two groups according to their gender ( 25 male and 50 female) all of them are suffering from major and severe Aphthous ulcers in the mouth. The results showed a decrease in the size of ulcer when the concentration of (AgNPs) solution was (10 $\mu \mathrm{g} / \mathrm{ml})$ figure (3). While, It was found that the increasing concentration of $(\mathrm{AgNPs})$ to $(20 \mu \mathrm{g} / \mathrm{ml})$ resulted in a highly significant decrease in aphthous ulcer size with a measurable zone of inhibition in both groups female and male patients during the first 24 hours figure (4) and table 1.

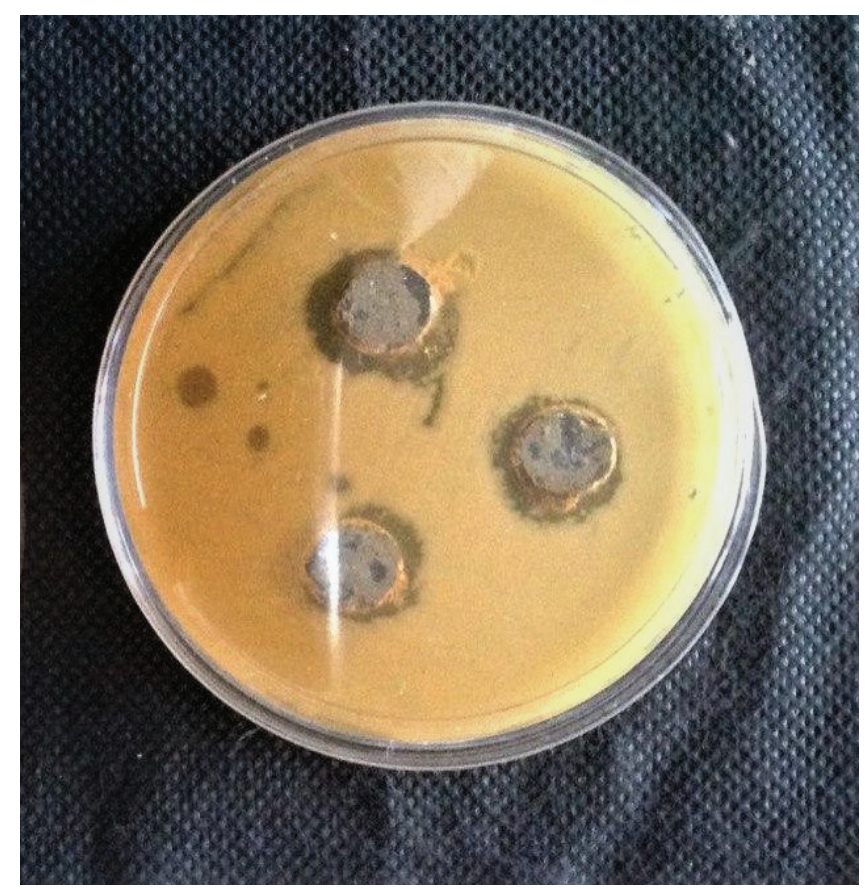

Figure (3): Inhibitory effect of silver nanoparticle solution at concentration of (10 $\mu \mathrm{g} / \mathrm{ml})$. 


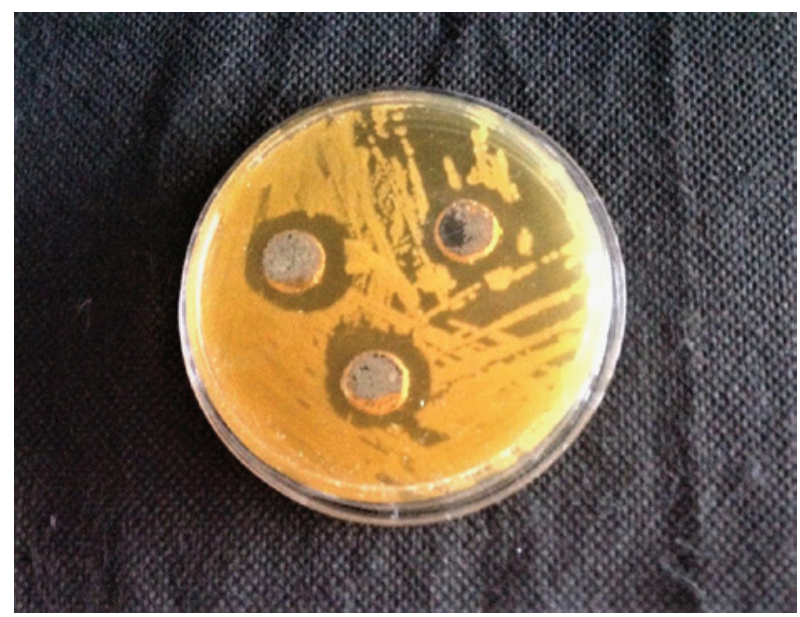

Figure (4): Inhibitory effect of silver nanoparticle solution at concentration of $(20 \mu \mathrm{g} / \mathrm{ml})$.

Table (1): Average Inhibitory zone level of different concentration of silver nanoparticles against aphthous stomatitis.

\begin{tabular}{|l|l|l|}
\hline Gender & $(\mathbf{1 0} \boldsymbol{\mu g} / \mathbf{m l}) \mathbf{A g N P s}$ & $\mathbf{( 2 0} \boldsymbol{\mu g} / \mathbf{m l}) \mathbf{A g N P s}$ \\
\hline Female & $0.35 \pm 0.10 \mathrm{~cm}$ & $1.1 \pm 0.20 \mathrm{~cm}$ \\
\hline Male & $0.45 \pm 0.13 \mathrm{~cm}$ & $1.4 \pm 0.16 \mathrm{~cm}$ \\
\hline
\end{tabular}

Silver nanoparticles have proven in many recent studies to exert a biological activity at non-cytotoxic concentrations, but the mechanism underlying their inhibitory activity has not been not fully elucidated (10). Nano sized silver particles is unlike other silver precursors like silver oxide and silver nitrate because of their enhanced surface area provides the more toxic effect to the microbes ${ }^{(6)}$.

A recent study by (Reidy et al.) (11), suggested that the Reactive Oxygen Species (ROS) released by the silver nanoparticles has been preventing the DNA replication, uncontrolled oxidation of proteins, breakdown of membrane function.

The U.S. Environmental Protection Agency has published a reference dose (Rfd), which is for oral silver exposure is $5 \mu \mathrm{g} / \mathrm{kg}$ daily, with a critical dose estimated at $14 \mu \mathrm{g} / \mathrm{kg}$ daily for the average person ${ }^{(1)}$. It has been reported that silver nanoparticles (SNPs) are nontoxic to humans and most effective against bacteria, viruses and other eukaryotic microorganisms at low concentrations and without any side effects ${ }^{(10)}$.

The limitation faced in our study was the inability to measure the size and shape of silver nanoparticles due to lack of measurement devices and also the high cost.

\section{Conclusion}

Silver nanoparticles (AgNPs) were synthesized using oxidation reduction method, characterization of resulting colloidal AgNPs by FTIR. Aphthous stomatitis is a common and recurrent painful disease of the oral mucous membrane. In this study, silver nanoparticles are showing promising inhibitory effect against Aphthous stomatitis. Moreover, It was found that Silver nanoparticles solution of $(20 \mu \mathrm{g} / \mathrm{ml})$ concentration resulted in a highly significant decrease in the size with a measurable zone of inhibition in both groups female and male patients during the first 24 hours in the cultured samples that isolated from Aphthous stomatitis. Further work is mandatory to investigate the inhibitory mechanism of (AgNPs) against Aphthous stomatitis and also to overcome the lack of measurement devices faced our current study.

Ethical Clearance: The Research Ethical Committee at scientific research by ethical approval of both $\mathrm{MOH}$ and MOHSER in Iraq

\section{Conflict of Interest: Non}

Funding: Self-funding

\section{References}

1. Alidaee MR, Taheri A, Mansoori P, Ghodsi SZ. Silver nitrate cautery in aphthous stomatitis: a randomized controlled trial. Br J Dermatol. 2005;153:521-525.

2. Gudikandula K, Charya Maringanti S. Synthesis of silver nanoparticles by chemical and biological methods and their antimicrobial properties. J. Exp. Nanosci. 2016;11(9):714-721.

3. Kim SW, Kim KS, Lamsal K, Kim YJ, Kim SB, Jung M, Sim SJ, Kim HS, Chang SJ, Kim JK, et al. An in vitro study of the antifungal effect of silver nanoparticles on oak wilt pathogen Raffaelea sp. J Microbiol Biotechnol. 2009;19(8):760-764.

4. Galdiero S, Falanga A, Vitiello M, et al. Silver nanoparticles as potential antiviral agents. Molecules. 2011;16:8894-8918. 
5. Babaee N, Zabihi E, Mohseni S, Moghadamnia A A. Evaluation of the therapeutic effects of Aloe vera gel on minor recurrent aphthous stomatitis. Dent Res J (Isfahan). 2012;9(4):381-5.

6. Zarei M, Jamnejad A, Khajehali E. Antibacterial effect of silver nanoparticles against four foodborne pathogens. Jundishapur J Microbiol. 2014;7(1):e8720. doi:10.5812/jjm.8720.

7. Wayne PA. Clinical and Laboratory standards Institute, “ Performance standards for Antimicrobial Susceptibility Testing. Inform Suppl. 2011;31(1):100-121.

8. Salar RK, Sharma P, Kumar N. Enhanced antibacterial activity of streptomycin against some human pathogens using green synthesized silver nanoparticle. Res. Eff. Technol (2015);1(2): 106115.

9. El-Batal AI, Ahmed SF. Therapeutic effect of Aloe vera and silver nanoparticles on acid-induced oral ulcer in gamma-irradiated mice. Braz oral res .2018;32:e004.

10. Lara HH, Turrent LI, Trevino EN. and Padilla CR. PVP-coated silvernanoparticles block the transmission of cell-free and cell-associated HIV1 in human cervical culture. J Nanobiotechnol. 2010; 8 (15):15-25.

11. Reidy B, Haase A, Luch A, Dawson KA and Lynch I. Mechanisms of Silver Nanoparticle Release, Transformation and Toxicity: A Critical Review of Current Knowledge and Recommendations for Future Studies and Applications. Materials. 2013; 6(6):2295-2350. 